The Astrophysical JouRnAL, 530:478-490, 2000 February 10

(c) 2000. The American Astronomical Society. All rights reserved. Printed in U.S.A.

\title{
A GENERALIZED MODEL FOR THE PROTON EXPANSION IN ASTROPHYSICAL WINDS. III. THE COLLISIONAL TRANSFERS AND THEIR PROPERTIES
}

\author{
FranÇOIS LeblanC AND DaNIEL Hubert \\ Département de Recherche Spatiale, CNRS UMR 8632, Observatoire de Paris, 92195 Meudon Cedex, France; francois.leblanc@obspm.fr \\ AND \\ PIERRE-LOUIS BLELLY \\ Centre d'Etude Spatiale des Rayonnements, CNRS UPS, 9 Avenue du Colonel Roche, BP 4346, F-31028 Toulouse Cedex 4, France \\ Received 1999 January 28; accepted 1999 September 29
}

\begin{abstract}
This is the third and last of a series of papers that present a new theoretical approach to modeling the expansion of the solar and terrestrial polar winds by solving the Fokker-Planck equation. The Coulomb collisional transfers between the different species that compose these winds are presented after the velocity distribution function and the set of transport equations associated with the generalized model. The method and the assumptions used to calculate these terms are described. They are derived from generic expressions, which must be numerically estimated for specific applications. Their new properties are analyzed in the context of the terrestrial polar wind, and their potential importance in the processes of heating and acceleration of the solar wind is discussed. Because the generalized model is adapted to reproduce the high suprathermal part of the velocity distribution function currently observed in the solar wind, we also emphasize the role of this contribution in the collisional transfers. In the terrestrial polar wind, the region of energy transfer between $\mathrm{H}^{+}$and $\mathrm{O}^{+}$ions is thinner than previously predicted. In the solar wind, the region of energy transfer between electrons and protons in the inner corona is larger than previously thought. The role of the thermal and mirror forces and the mechanisms of isotropization of the electrons are underlined.
\end{abstract}

Subject headings: atomic processes — interplanetary medium — plasmas — solar wind stars: winds, outflows

\section{INTRODUCTION}

The solar and terrestrial polar winds, called the astrophysical winds, have a common property; they escape from a collision-dominated region and reach a collisionless region despite the effect of a gravity field. Usually, we consider that two dependent phenomena must be understood. First, the original mechanisms, that generate the observed speed at $10 R_{\odot}$ for the fast solar wind or the supersonic speed of the $\mathrm{H}^{+}$ions in the terrestrial polar wind at an altitude of $4000 \mathrm{~km}$; and second, the evolution of the particle state during their expansion. In this set of papers, we have decided to deal with the second phenomenon, developing an original solution of the Fokker-Planck equation adapted to the observed nonequilibrium state.

There exists no available theory that is able accurately to model the evolution of a plasma from collision-dominated regions to collisionless regions. Indeed, there exist models that neglect the collisional effect (Chamberlain 1960; Lemaire \& Scherer 1973) and models for collisiondominated plasmas (Parker 1958; Banks \& Holzer 1968), but no model well adapted to the transition between these two situations. However, a third class of models, the multimoment approach (Grad 1958; Demars \& Schunk 1979), is currently more and more frequently used for the solar and terrestrial polar winds. It has already provided a welladapted description of the terrestrial polar wind (Ganguli 1996) and has significantly improved, with respect to recent observations, the classical fluid description of the acceleration of the solar wind by the description of the electron and proton heat flux (Olsen \& Leer 1996, 1999; Li 1999). The multimoment approach is an extension of the classical magnetohydrodynamical models to situations far from the local thermodynamic equilibrium state. It is a perturbative method that describes the state of the plasma with new macroscopic parameters related to those used in a hydrodynamic description; e.g., a temperature anisotropy, a stress tensor, or a heat flux. But these methods are finally limited to states near local equilibrium, that is, in the context of the wind expansion, to small values of the rate between the heat flux and the free-streaming heat flux. Indeed, in Leblanc \& Hubert (1997), we have shown that the approaches deduced from the Grad model have difficulties describing most of the characteristics of the proton velocity distribution function observed in the solar wind by Helios 1 and 2 (Marsch et al. 1982a, 1982b).

The generalized model is also a multimoment approach, but devoted to describing states far from local equilibrium. Leblanc \& Hubert $(1997,1998)$ have shown that this new approach is better adapted to the typical in situ observations made in the solar wind. Indeed, the microscopic description is a good approximation of the observations of the distribution function. The truncation of the set of transport equations is in good agreement with the typical observations in the solar wind between 0.3 and $1 \mathrm{AU}$ (Leblanc \& Hubert 1997). The velocity distribution function never developed negative values, contrary to the previous multimoment approaches applied to model the astrophysical winds. The macroscopic description respects the hyperbolicity and realizability criteria for constructing the set of transport equations (Leblanc \& Hubert 1998), and it is able to reproduce the typical profile of the macroscopic parameters in the terrestrial polar wind (Leblanc, Hubert, \& Blelly 2000). At least the generalized model accounts better for the suprathermal particles than the other multimoment models. 
In this paper we present the Coulomb collisional transfers between species calculated in the frame of the generalized model. This paper is a first analysis of the role, in the interaction processes, of the suprathermal part of the velocity distribution that is observed in the solar wind between 0.3 $\mathrm{AU}$ and several AU for protons and $\alpha$ ions (Marsch et al. 1982a, 1982b), for the electrons (Pilipp et al. 1990), and for minor ions in the solar corona between 1 and $10 R_{\odot}(\mathrm{Kohl}$ et al. 1997). We have no direct measurements of the distribution function in the terrestrial polar wind, but all the theoretical models predict such a suprathermal part.

Section 2 gives a brief summary of the method used to obtain the collisional transfer expressions. We also describe the assumptions made for applications to the solar and terrestrial polar winds. In the $\S 3$, these new expressions are analyzed in the context of the terrestrial polar wind and in the context of the solar wind in the lower part of the solar corona. Our conclusions are presented in $\S 4$. The expressions for the complete set of transport equations are presented in Appendices A and B.

\section{THE COLLISIONAL TRANSFERS}

A multimoment approach is based on the assumption that the microscopic state can be accurately described using a finite number of velocity moments of the velocity distribution function. A velocity moment is defined as

$$
\left\langle C_{s}^{p}\right\rangle=\frac{1}{n_{s}} \int_{V} f_{s}\left(r, v_{s}, t\right) C_{s}^{p} d c_{s},
$$

where $p$ is the order of the velocity moment, $n_{s}$ is the number density of the species $s, f_{s}$ is the velocity distribution of the species $s, c_{s}=\boldsymbol{v}_{s}-\boldsymbol{u}_{s}$ is the peculiar velocity, $\boldsymbol{v}_{s}$ is the species velocity, $\boldsymbol{u}_{s}$ is the average velocity of the species, and $\boldsymbol{r}$ is the spatial position. $C_{s}^{p}$ is a tensor product of $p$ terms of components $\left(c_{s 1}, c_{s 2}, c_{s 3}\right)$, where the numbers 1,2 , and 3 indicate a particular direction in the velocity space, $V$. The velocity moments, $\left\langle C_{s}^{p}\right\rangle$, are linked to the classical macroscopic parameters (Leblanc \& Hubert 1998).

The velocity moments are determined by solving the transport equations. These equations are obtained by the integration of a kinetic equation, which could be the Boltzmann equation or the Fokker-Planck equation. This operation can be schematized by

$$
\int_{V} C_{s}^{p}\left\{\frac{\partial f_{s}}{\partial t}+\left(\boldsymbol{c}_{s}+\boldsymbol{u}_{s}\right) \cdot \frac{\partial f_{s}}{\partial \boldsymbol{r}}+\frac{\boldsymbol{F}_{0}}{m_{s}} \cdot \frac{\partial f_{s}}{\partial \boldsymbol{c}_{s}}=C\left(f_{s}, f_{t}\right)\right\} d \boldsymbol{c}_{s},
$$

which represents the evolution of the velocity moments $\left\langle C_{s}^{p}\right\rangle$ as a function of the time $t$ and the space $r$. In equation (2), $\boldsymbol{F}_{0}$ is the sum of all the exterior forces and $m_{s}$ the mass of the particles of the $s$ species. $C\left(f_{s}, f_{t}\right)$ gives the change in the $s$ species due to encounters of $s$ particles with particles of type $t$. The technique for constructing these equations is described in Jancel \& Kahan (1963), Tanenbaum (1967), and Burgers (1969). We have chosen to integrate the Fokker-Planck equation, which is well adapted to describing the Coulomb interactions between the particles that compose the astrophysical winds.

In such a case, the collisional transfer $\int_{V} C_{s}^{p}\left\{C\left(f_{s}, f_{t}\right)\right\} d c_{s}$ cannot be determined without an a priori form of the two distributions $f_{s}$ and $f_{t}$. A direct relation between the velocity distribution function and the velocity moments that are used as unknowns is needed. This relation allows us to close the set of transport equations. For instance, Grad (1958) described the distribution function by a polynomial development based on a local Maxwellian function. He developed models for $5,8,13$, and 20 independent velocity moments. Oraevskii, Chodura, \& Feneberg (1968), Chodura \& Pohl (1971), and then Demars \& Schunk (1979) have considered situations that could be described by a polynomial development based on a bi-Maxwellian function. They improved Grad's description for situations in which high values of the temperature anisotropy could exist. They also defined the 16 velocity moments that are needed to properly describe the expansion of the solar and terrestrial polar winds. These 16 parameters correspond to the three first orders of the velocity moment (for $p=0-3$ ) in a gyrotropically dominated situation. In Leblanc \& Hubert (1997), we presented and discussed a new choice of distribution function (see eqs. [A2] and [A3]). We made this choice to better take into account the suprathermal part of the velocity distribution, which could play an important role in the processes of acceleration of the particles in astrophysical winds.

The left-hand side of equation (2) was presented and discussed in Leblanc \& Hubert (1998). In this paper we present and analyze the properties of the collisional terms deduced from the right-hand side of equation (2). The expressions of $f_{s}$ and $f_{t}$ allow us to determine an exact form for the collisional transfers associated with each velocity moment. In the context of further applications to the astrophysical winds, we have chosen the 16 independent parameters of Demars \& Schunk (1979), which are defined in Leblanc \& Hubert (1998).

Because of the high complexity of the collisional transfer, Burgers (1969) defined a semilinear approximation in the case of the Grad approach. This approximation is based on the assumption that the different species are sufficiently collisional to remain not far from the local thermodynamic equilibrium state; the distribution function of each species is supposed to be not far from a Maxwellian distribution, and thus the corrections to this function should not be important. If the expression of the velocity distribution $f_{s}$ or $f_{t}$ in a multimoment approach is schematized by $f=g^{0}(1+\phi), g^{0}$ is then called the zeroth-order approximation and $\phi$ the polynomial part. The term $g^{0}$ is a local Maxwellian function in the Grad approach and a biMaxwellian function in the Chodura \& Pohl (1971) and Demars \& Schunk (1979) approaches. In the semilinear approximation, the polynomial part, $\phi$, should be small with respect to 1 because we consider states that could be describe approximately by $g^{0}$. Therefore, when determining the collisional transfer, the product $f_{s} f_{t}$ is given by $f_{s} f_{t} \simeq$ $g_{s}^{0} g_{t}^{0}\left(1+\phi_{s}+\phi_{t}\right)$, which shows that $\phi_{s} \phi_{t}$ has been neglected with respect to 1 . On the other hand, the parameters that characterize the difference from the local equilibrium state, such as the stress tensor or the heat flux, are supposed to be close to zero. Burgers (1969) also supposed that the relative velocity between two species is always smaller than the thermal velocity,

$u_{s}-u_{t} \ll v_{s t, \mathrm{th}}, \quad$ where $\quad v_{s t, \mathrm{th}}=\left(\frac{2 k_{\mathrm{B}} T_{s}}{m_{s}}+\frac{2 k_{\mathrm{B}} T_{t}}{m_{t}}\right)^{1 / 2}$,

where $k_{\mathrm{B}}$ is the Boltzman constant and $T_{s}$ is the temperature of the $s$ species. He then neglected all the contributions in the collisional transfers that depend on the product of the 
relative velocity by a term of the stress tensor or a term of the heat flux. As an example, the semilinear approximation neglects all the terms proportional to $\left(u_{s}-u_{t}\right)^{p} / v_{s t, t h}^{p}$ where $p \geq 2$.

However, because the generalized model is built to consider states that could be far from the local thermodynamic equilibrium, we change some of these assumptions. As previously, the correction term $\phi$ to the zeroth-order function is supposed to be small with respect to 1 . In the perpendicular direction, the relative velocity between two species is supposed to be much smaller than the thermal velocity, while this assumption is not made for the parallel direction. Indeed, observations in the solar wind (Marsch et al. 1982a, $1982 \mathrm{~b}$ ), as well as observations and theoretical models of the terrestrial polar wind (Abe et al. 1993; Blelly \& Schunk 1993) have indicated that the relative speed in the direction parallel to the magnetic field could be of the order of the thermal velocity. To sum up, we applied the semilinear approximation for the perpendicular direction but kept all the contributions that depend on the parallel direction in the expressions of the collisional transfer.

In this paper we consider a one-dimensional situation; that is, we suppose that the plasma is gyrotropically dominated by the strong magnetic field. The motion of the particles is then confined along the magnetic field lines. This assumption greatly simplifies the set of transport equations. From the 16 independent parameters considered in Leblanc \& Hubert (1998), we only keep six parameters, described in Appendix A (see eq. [A1]), which are referred to as the six moments. The expressions of the collisional transfers are presented in Appendix B. Comparing them to the similar expressions of Demars \& Schunk (1979), we can remark that the new expressions (see eqs. [B3]-[B6]) generalize these terms. As explained in Leblanc \& Hubert (1997), the model of velocity distribution functions contains the biMaxwellian function of Demars \& Schunk (1979). The expressions of Demars \& Schunk (1979) are then found again when the parameters $D_{s}^{*}$ and $D_{t}^{*}$ in equations (B3)(B6) are set to zero.

\section{PROPERTIES OF THE COLLISIONAL TRANSFERS}

The choice to develop a multimoment and multispecies approach is motivated by an analysis of the observations made in the solar and terrestrial polar winds. For instance, observations have shown that the mechanisms of heating and acceleration of the solar wind are different for the different species (electrons, protons, and $\alpha$ ions), and that the interactions between species could play an important role. The most recent models of these winds are then based on multifluid descriptions (see Olsen \& Leer 1996, 1999; Tu \& Marsch 1997; Li, Esser, \& Habbal 1997; Li 1999) and have underlined the importance of heat transfers (Olsen \& Leer 1996; Tu \& Marsch 1997) and the role of heavy ions (Li et al. 1997; Kohl et al. 1998). The temperature anisotropy should also be considered as a potential mechanism for acceleration of the particles via the mirror force, as has already been emphasized for the terrestrial polar wind (Jones et al. 1998). Therefore, in a theoretical approach it is important to integrate an accurate description of the collisional processes, of the transport of the temperature anisotropy, and, as a consequence, of the transport of the heat-flux anisotropy. This is why we derived an analytic formulation of the collisional transfers despite the difficulty. This work was done for each velocity moment correspond- ing to the density, velocity, temperature, and heat flux of the different species.

With the new expressions for the collisional transfers, we are not able to directly identify the main contributions of the collisional transfer as thermal and friction forces in the momentum transfer (eq. [B3]) or thermalization and friction terms in the energy transfer (eqs. [B4] and [B5]). However, we can numerically estimate the role of these terms from typical profiles of the macroscopic parameters in these winds and compare the new collisional transfers to those deduced from the previous multimoment and multispecies models (Demars \& Schunk 1979; Chodura \& Pohl 1971). In the next sections, we will consider the collisional transfers associated with the 16 moment approximation deduced from the work of Demars \& Schunk (1979) for the momentum and heat-flux transfer, and the more complete expressions of Chodura \& Pohl (1971) for the energy transfer.

\subsection{In the Terrestrial Polar Wind}

Although the generalized model was firstly developed in the context of the solar wind, we have shown in previous papers (Leblanc \& Hubert 1997, 1998) that this model is also well adapted to the description of the terrestrial polar wind.

Figures 1-5 are deduced from theoretical profiles of the six velocity moments determined by Blelly \& Schunk (1993) for electrons and $\mathrm{H}^{+}$and $\mathrm{O}^{+}$ions in the terrestrial polar wind at altitudes between 200 and $8000 \mathrm{~km}$. These authors have compared different multimoment approaches based on Maxwellian distribution functions (5, 8, and 13 moments; Schunk 1977) and on a bi-Maxwellian distribution function (16 moments; Demars \& Schunk 1979). These profiles were obtained with a gyrotropically dominated 16 moment bi-Maxwellian approach in the case of a stable neutral atmosphere with an electron solar extremeultraviolet (EUV) heating, simulated by an electron heat flow imposed at the top boundary and dissipated by collision with neutrals and ions. The result of this simulation is a supersonic flow of $\mathrm{H}^{+}$ions and a subsonic flow of $\mathrm{O}^{+}$ions escaping from the terrestrial polar ionosphere. At an altitude of $8000 \mathrm{~km}$, the $\mathrm{H}^{+}$velocity is of the order of $10 \mathrm{~km}$ $\mathrm{s}^{-1}$, and for $\mathrm{O}^{+}$ions it is of the order of $1 \mathrm{~km} \mathrm{~s}^{-1}$, which is in good agreement with in situ measurements (Abe et al. 1993). The temperatures reach a maximum of $6000 \mathrm{~K}$ for $\mathrm{H}^{+}$ions around $4000 \mathrm{~km}$ and $3500 \mathrm{~K}$ for $\mathrm{O}^{+}$ions around $2000 \mathrm{~km}$. The increase of the electron temperature is roughly constant and reaches $10,000 \mathrm{~K}$ around $8000 \mathrm{~km}$, with a very weak anisotropy. Afterward, we will only describe the interaction of the $\mathrm{H}^{+}$ions with the electrons and the $\mathrm{O}^{+}$ions.

In Figure 1, the left panel displays the relative velocity between $\mathrm{H}^{+}$and electrons (dotted line) and between $\mathrm{H}^{+}$and $\mathrm{O}^{+}$ions (solid line), normalized to the thermal velocity as defined in equation (3). The right panel displays the normalized total heat flux for each species $\left(\mathrm{H}^{+}\right.$ions, solid line; $\mathrm{O}^{+}$ ions, dotted line; and electrons, dashed line). The normalized heat flux is defined as the heat flux normalized by the freestreaming heat flux: $n_{s} m_{s}\left(k_{\mathrm{B}} T_{s} / m_{s}\right)^{3 / 2}$. The normalized heat flux is always smaller than the limit of validity determined in Leblanc \& Hubert (1998). The transfers between $\mathrm{H}^{+}$ions and electrons will not be considered. Indeed, the relative velocity between these two species and the normalized heat flux for the electrons is always small, because of the very 


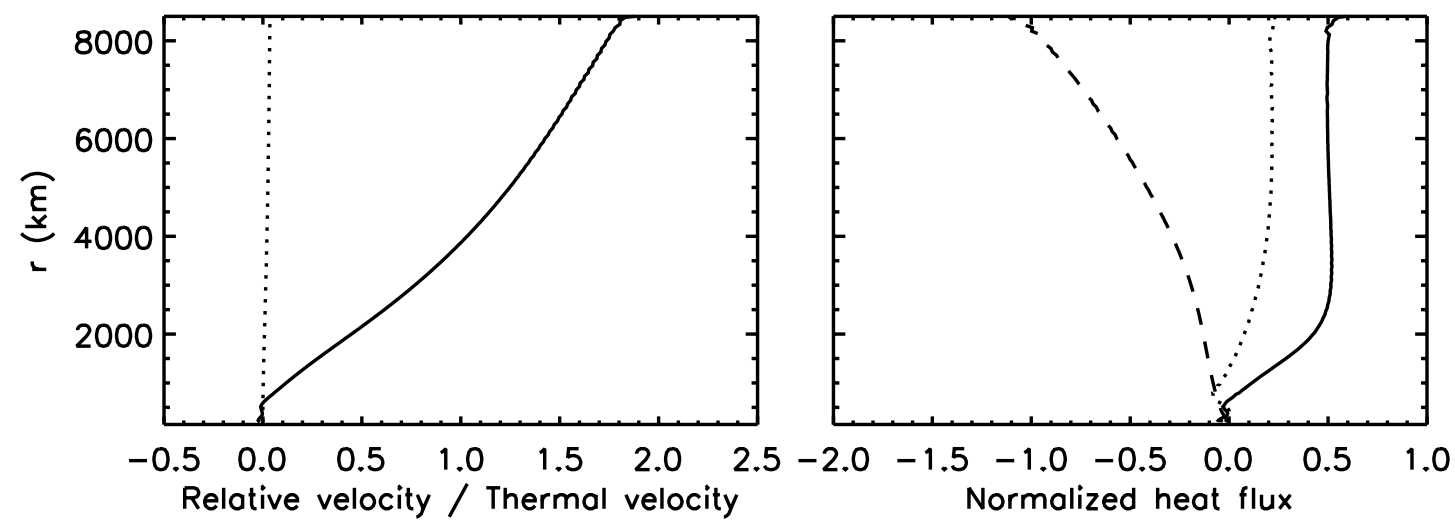

FIG. 1.-Left: Relative velocity between $\mathrm{H}^{+}$and $\mathrm{O}^{+}$ions (solid line) and between $\mathrm{H}^{+}$ions and electrons (dotted line) as functions of the altitude, normalized to the thermal velocity determined from the thermal velocities of both considered species (see eq. [3]). Right: Heat flux of parallel energy for $\mathrm{H}^{+}$ ions (solid line), $\mathrm{O}^{+}$ions (dotted line), and electrons (dashed line) as functions of the altitude, normalized to the free-streaming heat flux associated with each species. These profiles are derived from typical profiles of the six velocity moments in the terrestrial polar wind (Blelly \& Schunk 1993).

large electron thermal velocity. As a consequence, the differences between the generalized and the bi-Maxwellian models are not important. On the other hand, the relative velocity between $\mathrm{H}^{+}$and $\mathrm{O}^{+}$ions is not negligible. We then observe significant differences between the two models.

In the momentum transfer, an important contribution is the friction force, $v_{s t}\left(u_{t}-u_{s}\right)$, where $v_{s t}$ is the collision frequency of the particles of the $s$ species with the particles of the $t$ species. A second important term is the thermal force, which depends on the heat flux: $v_{s t}\left(q_{s} / \rho_{s}-q_{t} / \rho_{t}\right) / v_{s t \text { th }}^{2}$, where $v_{s t, \mathrm{th}}^{2}=v_{s, \mathrm{th}}^{2}+v_{t, \mathrm{th}}^{2}, v_{s, \mathrm{th}}=\left(2 k_{\mathrm{B}} T_{s} / m_{s}\right)^{1 / 2}$ is the thermal velocity of the $s$ species, $\rho_{s}=n_{s} m_{s}$ is the mass density, and $q_{s}$ is the total heat flux of the $s$ species. The thermal force is a correction of the friction force that takes into account the dependence of the collision frequency as a function of the relative velocity for Coulomb collisions. For a pure Maxwellian potential interaction, this contribution does not appear. In Figure 2 we present the momentum transfer of the generalized approach (solid line), the friction force

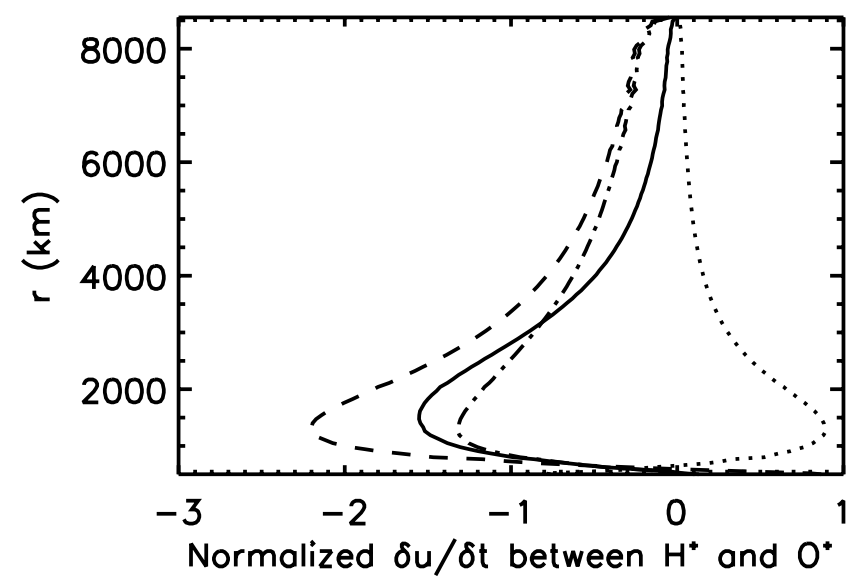

FIG. 2.-Comparison of the momentum-generalized transfer (solid line) between $\mathrm{H}^{+}$and $\mathrm{O}^{+}$ions for typical profiles of the six velocity moments in the terrestrial polar wind (derived from Blelly \& Schunk 1993) with the friction force (dashed line), the thermal force (dotted line), and the sum of these two forces (dash-dotted line). These transfers are normalized to the electron pressure force. (dashed line), the thermal force (dotted line), and the sum of the friction and thermal forces (dash-dotted line) between $\mathrm{H}^{+}$and $\mathrm{O}^{+}$ions. These parameters are normalized to the electron pressure force, $1 / \rho_{e} \partial\left(n_{e} k_{\mathrm{B}} T_{e}\right) / \partial r$, which is equal to the effect of the charge-separation electrostatic field $E$ (see Appendix A). This term is usually identified as one of the main acceleration mechanisms of the particles. The effect of the interaction between the $\mathrm{H}^{+}$and $\mathrm{O}^{+}$ions is to slow down the $\mathrm{H}^{+}$ions, which move faster than the $\mathrm{O}^{+}$ions. Figure 2 indicates that the friction force is always dominant in the collisional momentum transfer. The thermal force is opposite in sign to the friction force. The sum of the friction and thermal forces in the dash-dotted line is never negligible with respect to the charge-separation electrostatic field $E$. The generalized transfer is not exactly the sum of these two terms, in particular in the region of acceleration of the particles, mainly below $2000 \mathrm{~km}$. The generalized model predicts a slowing down of the $\mathrm{H}^{+}$ions due to interaction with the $\mathrm{O}^{+}$ions that is more important than the effect of the sum of the thermal and friction forces. We can conclude that the suprathermal part of the velocity distribution function, which is better taken into account by the generalized model than by the bi-Maxwellian model, plays a nonnegligible role in the acceleration process of the particles (in this particular application).

We can identify three contributions in the energy transfer of Chodura \& Pohl (1971). The first is the thermalization term, $m_{s} v_{s t}\left(T_{s}-T_{t}\right) /\left(m_{s}+m_{t}\right)$; the second is a friction term, $m_{s} v_{s t}\left(u_{s}-u_{t}\right)^{2} / k_{\mathrm{B}} ;$ and the third term, $2 m_{s} v_{s t}\left(u_{s}-u_{t}\right)\left(-q_{s} / \rho_{s}\right.$ $\left.+q_{t} / \rho_{t}\right) /\left(v_{s t, \mathrm{th}}^{2} k_{\mathrm{B}}\right)$, could be understood as a correction term to the friction, which takes into account the dependence of the collision frequency as a function of the relative velocity. This term does not appear in the expression of Demars \& Schunk (1979) because of the assumption of a low parallel relative velocity between species with respect to the thermal velocity and of a low total heat flux with respect to the free-streaming heat flux. Chodura \& Pohl (1971) did not make this assumption. In Figure 3 we present the total energy transfer of the generalized approach (solid line), the sum of the friction and thermalization terms (dashed line), the correction to the friction term (dotted line), and the sum of these two last contributions (dash-dotted line). The total energy transfer is defined as $\delta p_{s} / \delta t=\left(\delta p_{s \|} / \delta t+2 \delta p_{s \perp} / \delta t\right) / 2$, 


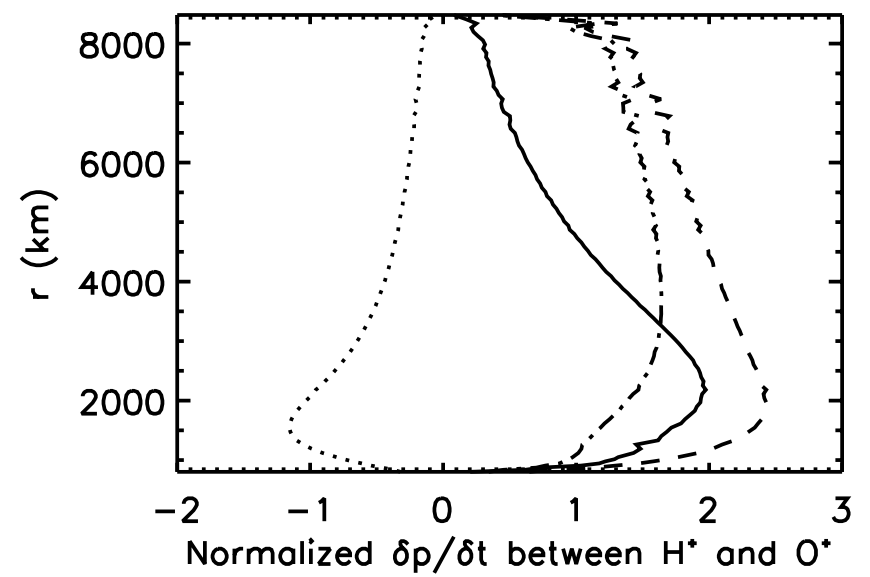

FIG. 3.-Comparison of the generalized transfer of total energy (solid line) between $\mathrm{H}^{+}$and $\mathrm{O}^{+}$ions for typical profiles of the six velocity moments in the terrestrial polar wind (derived from Blelly \& Schunk 1993) with the sum of the thermalization and friction terms (dashed line), the correction of the friction term (dotted line), and the sum of these two contributions (dash-dotted line). These transfers are normalized as explained in $\S 3.1$.

where $\delta p_{s \|} / \delta t$ and $\delta p_{s \perp} / \delta t$ are the parallel and perpendicular energy transfers, respectively (see eqs. [B4] and [B5] for the generalized model). The different contributions are normalized to the sum of two terms of the left-hand side of the equation for the total energy: $T_{\mathrm{H}^{+} \|} \partial u_{\mathrm{H}^{+}} / \partial r+\left[3 \partial q_{\mathrm{H}^{+}} / \partial r\right] /$ $\left(2 n_{\mathrm{H}^{+}} k_{\mathrm{B}}\right)$. In Figure 3, the friction and thermalization terms are always more important than the correction of the friction term and have opposite signs. This last term is mainly important in the acceleration and heating region of the particles, that is, between altitudes of 200 and $4000 \mathrm{~km}$. The friction and the thermalization terms dominate the equation for the total energy at almost all altitudes. Once again, it seems that the suprathermal part of the velocity distribution function plays an important role in the determination of the energy transfer. Indeed, if we compare the generalized energy transfer (solid line) with the sum of the three components (dash-dotted line), we remark that the difference between the two profiles is important. The generalized model predicts a higher increase of the $\mathrm{H}^{+}$temperature in the region of heating (for altitudes less than $3000 \mathrm{~km}$ ) and a lower increase at higher altitudes.

In order to complete the comparisons between the 16 moment bi-Maxwellian model and the generalized model, we also provide the normalized transfers of parallel and perpendicular energies and the nonnormalized transfers of heat flux for parallel and perpendicular energy. The normalization of the energy transfer is the same as in Figure 3. In Figure 4 we present the transfer of parallel (left) and perpendicular (right) energies between $\mathrm{H}^{+}$and $\mathrm{O}^{+}$ions for the generalized model (solid line) and for the bi-Maxwellian model (dotted line). The generalized transfer of energy predicts a higher variation of the anisotropy of the energy transfer than the bi-Maxwellian model. The parallel energy transfer also tends more rapidly to zero (for altitudes above $4000 \mathrm{~km}$ ). For the perpendicular part, the bi-Maxwellian transfer is always negative, whereas the perpendicular energy transfer predicted by the generalized model is positive above $1500 \mathrm{~km}$. The transfer of energy is more important for the parallel energy than for the perpendicular energy, according to the generalized model. The parallel temperature will then be higher than predicted by the biMaxwellian approach, in the acceleration region.

Figure 5 presents the profiles of the heat-flux transfer for the parallel (left) and perpendicular energy (right) compared with the 16 moment bi-Maxwellian expressions. The transfer of the heat flux is presented for the generalized model (solid line) and the bi-Maxwellian model (dotted line). Figure 5 shows that the transfers of the heat flux are similar in both approaches.

\subsection{In the Solar Wind}

We are not able to perform the same analysis in the solar wind, because we do not have theoretical profiles of the six velocity moments as in the terrestrial polar wind. However, we can make some remarks and predictions about the transfer between the different species in the solar wind. Here we only consider the two main species that compose the solar wind, that is, protons and electrons. We will restrict our analysis to the fast solar wind, which originates from the polar coronal holes. The collisional transfer for the slow

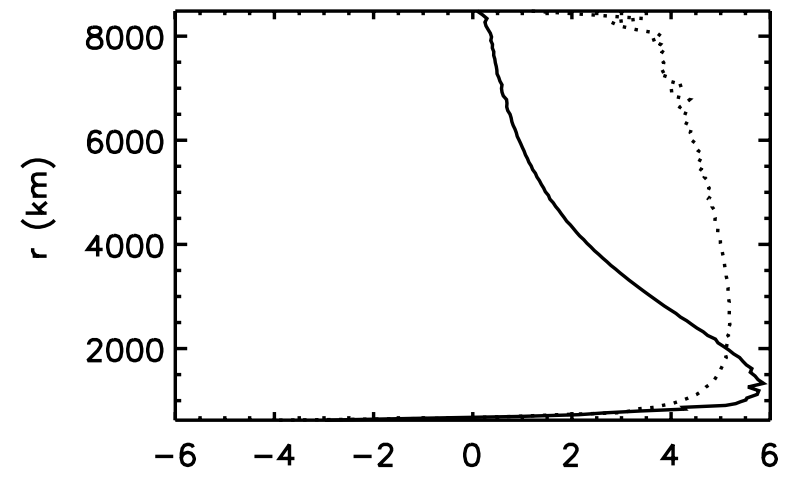

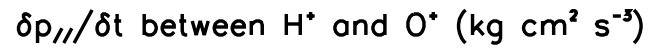

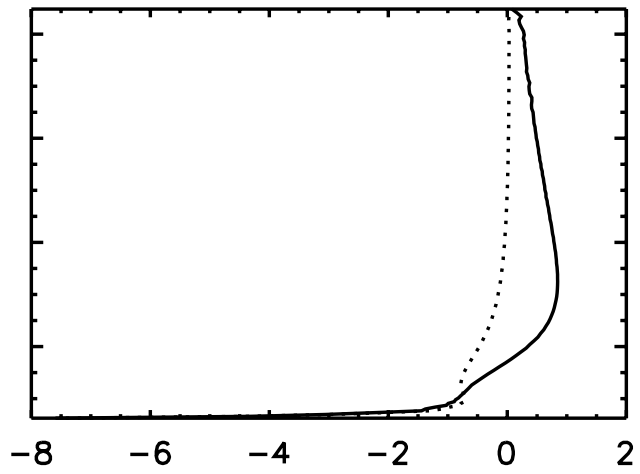

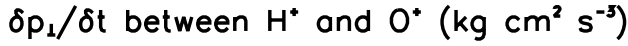

FIG. 4.- Perpendicular and parallel energy transfers between $\mathrm{H}^{+}$and $\mathrm{O}^{+}$ions as functions of the altitude. Collisional transfers are derived from the generalized model (solid line) and from the model of Chodura \& Pohl (1971; dotted line). Left: Parallel energy. Right: Perpendicular energy. These profiles are derived from typical profiles of the six velocity moments in the terrestrial polar wind (Chodura \& Pohl 1971) and have been normalized in the same way as for Fig. 3. 


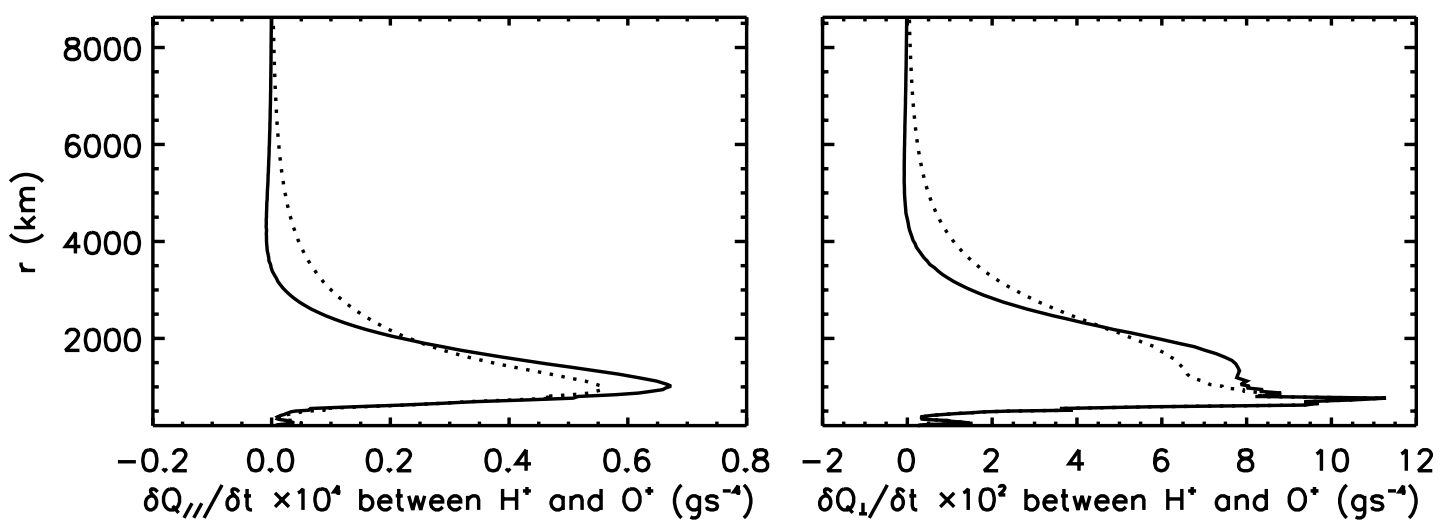

FIG. 5.- Transfer of heat flux between $\mathrm{H}^{+}$and $\mathrm{O}^{+}$ions as functions of the altitude. Collisional transfers are derived from the generalized model (solid line) and from the model of Demars \& Schunk (1979; dotted line). Left: Heat flux of parallel energy. Right: Heat flux of perpendicular heat flux. These profiles are derived from typical profiles of the six velocity moments in the terrestrial polar wind (Blelly \& Schunk 1993).

solar wind should be more important, due to the higher density of particles $\left(10\right.$ particles per $\mathrm{cm}^{3}$ at $1 \mathrm{AU}$ in the slow solar wind, whereas there are only 2 or 3 particles per $\mathrm{cm}^{3}$ in the fast solar wind). In this paragraph, we show how an accurate description of the collisional transfers between electrons and protons could help us to better understand the observed behavior of the electron velocity and temperature in the fast solar wind.

In Figure 6 we present the profiles of the density (top left), velocity (top right), and proton and electron temperatures (dotted and solid lines, respectively) in the bottom left panel for heliocentric distances between 1.1 and $10 R_{\odot}\left(1 R_{\odot}=1\right.$ solar radius). This region corresponds to the region of acceleration of the fast solar wind, according to Esser et al. (1997). The density profile is derived from measurements in the polar coronal hole reported by Tu \& Marsch (1997) and
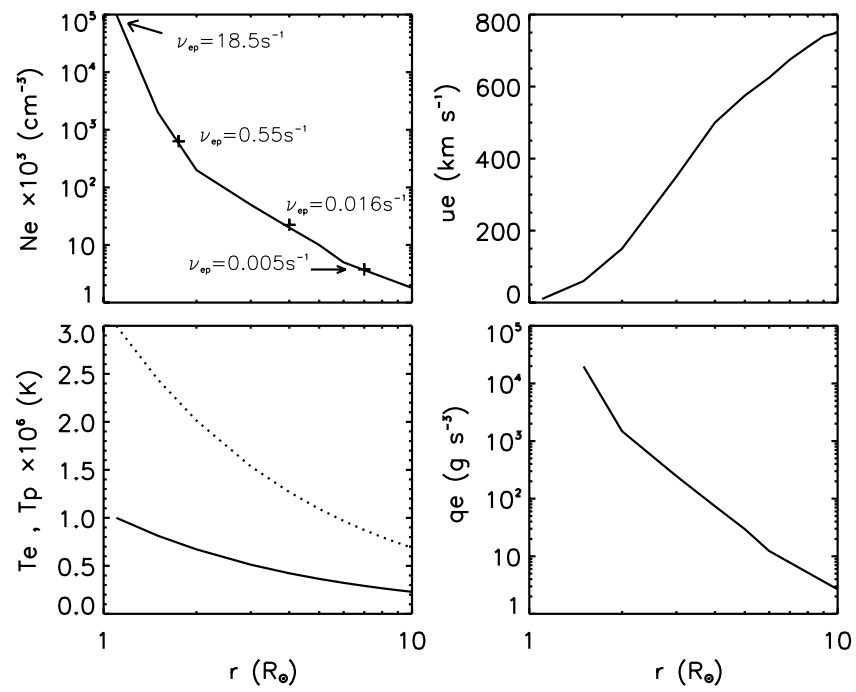

FIG. 6.-Top left: Profile of the electron density in the polar solar corona and Coulomb collision frequency, $v_{\text {ep }}$, of electrons with protons. Top right: Profile of the mean velocity of electrons in the solar corona (Esser et al. 1997). Bottom left: Profiles of the electron temperature (solid line) and of the proton temperature (dotted line), determined as explained in $\S$ 3.2. Bottom right: Total electron heat flux derived from the relation $q_{e}=0.25 n_{e} m_{e}\left(k_{\mathrm{B}} T_{e} / m_{e}\right)^{3 / 2}$. by Esser, Richard, \& Brickhouse (1998). The velocity profile is derived from the measurements made in the same region and reported by Esser et al. $(1997,1998)$. The proton and electron temperatures are set to $3 \times 10^{6}$ and $1 \times 10^{6}$, respectively, at $1.1 R_{\odot}$ (Kohl, Strachan, \& Gardner 1996; David et al. 1998) and are supposed to evolve between 1.1 and $10 R_{\odot}$ following a law in $r^{-2 / 3}$, where $r$ is the heliocentric distance. This law is inferred from observations beyond 0.3 AU (Marsch et al. 1982b; Issautier et al. 1998). The profiles of the electron density and temperature allow us to estimate the Coulomb collision frequency of the electrons with the protons. The collision frequency is roughly equal to $20 \mathrm{~s}^{-1}$ at $1.1 R_{\odot}$ for the interaction between electrons and protons and less than $0.001 \mathrm{~s}^{-1}$ at $10 R_{\odot}$ (see Fig. 6 , top left). Because the collision frequency of protons with electrons is much smaller than the collision frequency of electrons with protons, the interactions of electrons with protons are the main collisional effect in the corona. At the end, the processes of interaction should be only partial, because the collision frequency decreases rapidly between 1.1 and $10 R_{\odot}$, according to the mean velocity of the species in these regions (Esser et al. 1997, 1998). In the bottom right panel of Figure 6, we have plotted the total electron heat flux between 1.5 and $10 R_{\odot}$. The total electron heat flux is supposed to be proportional to $25 \%$ of the free-streaming heat flux. Indeed, we suppose that the total heat flux reaches its threshold of saturation, which has been observed in the solar wind between 1 and $5 \mathrm{AU}$ in the ecliptic plane (Scime et al. 1994) or predicted by experiments and plasma theories (Bell, Evans, \& Nicholas 1980). The temperature anisotropy is set to 1.2 for electrons and 1 for protons. The electron and proton heat-flux anisotropy is set to 1 . We verified that a proton temperature anisotropy in agreement with observations (Kohl et al. 1998) does not significantly change the results. The collisional transfers also depend on the relative velocity between electrons and protons. This relative velocity is difficult to estimate in the solar corona but could be proportional to the Alfvén speed, according to observations made at $1 \mathrm{AU}$ in the ecliptic plane (Feldman et al. 1976). Moreover, the SOHO Ultraviolet Coronagraph and Spectrometer (UVCS) observations (Kohl et al. 1997) have shown that the ions do not have the same velocity between 1 and $10 R_{\odot}$. Indeed, the $\mathrm{O}^{5+}$ ions reach a velocity of 400 

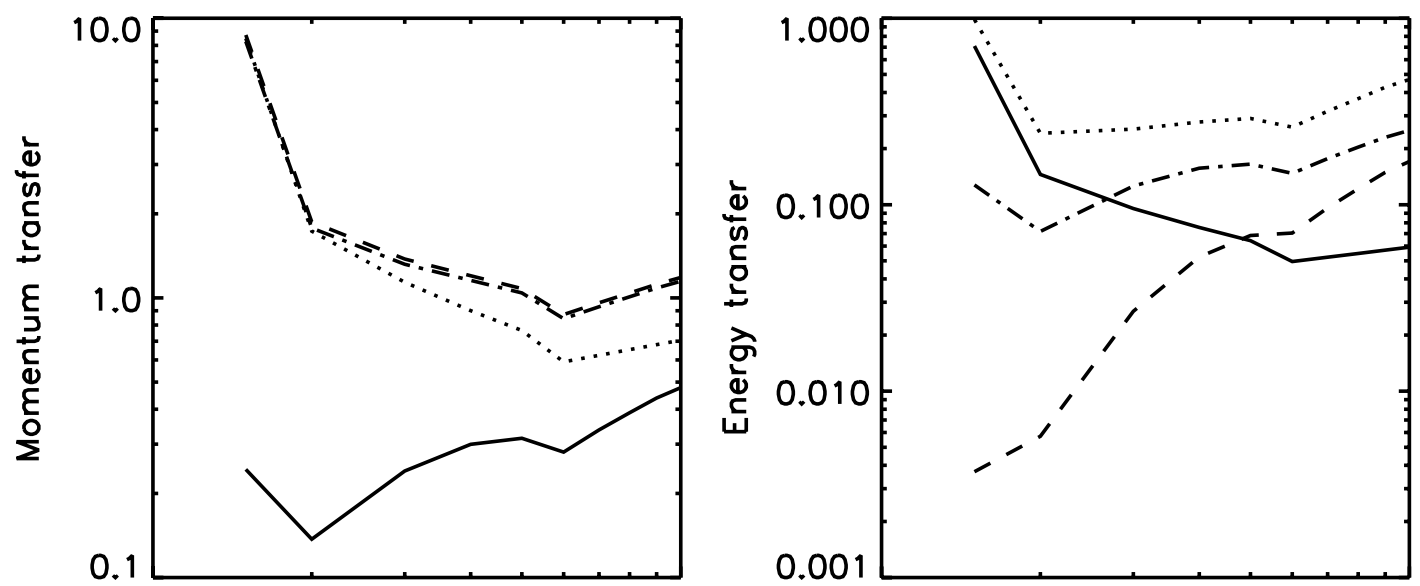

FIG. 7.-Left: Normalized friction force (solid line), thermal force (dotted line), generalized momentum transfer (dashed line), and bi-Maxwellian momentum transfer (dash-dotted line) in the solar corona derived from the temperatures, density, and heat flux given in Fig. 6. Right: friction term (dashed line), correction of the friction term (dash-dotted line), generalized total energy transfer (dotted line), and thermalization term (solid line), defined and normalized as described in $\S 3.2$.

$\mathrm{km} \mathrm{s}^{-1}$ at $2 R_{\odot}$ in the polar corona hole, whereas neutral $\mathrm{H}^{0}$, and then protons, reach a velocity of about $200 \mathrm{~km} \mathrm{~s}^{-1}$ at $2 R_{\odot}$. This observation could be explained by the fact that the mechanisms of heating and then of acceleration of the particles depend on the mass of the particles. It then seems realistic to suppose, because of these different ion velocities and the assumption of zero current, that there exists a relative velocity between electrons and protons in the region of heating and acceleration of these particles. We set the relative velocity between cold electrons and protons to be of the order of $25 \%$ of the mean velocity.

Moreover, according to Feldman (1976), the protons move faster than the core of the electron distribution and then accelerate the electrons of the core via the friction force. The thermal force has the same effect, if we consider the increase of the electron heat flux with the heliocentric distance starting from zero at $1.1 R_{\odot}$ in the collisiondominated region and reaching the positive and nonzero value observed at $0.3 \mathrm{AU}$ (Pilipp et al. 1990). Figure 7 (left) presents a possible evolution of these two forces derived from Chodura \& Pohl (1971). In this panel we have plotted the friction force (solid line), the thermal force (dotted line), the generalized momentum transfer (dashed line; see eq. [B3]), and the bi-Maxwellian momentum transfer (dashdotted line). The bi-Maxwellian momentum transfer is equal to the sum of the friction and thermal forces. All the contributions are normalized to the electron-pressure force $\left(1 / \rho_{e}\right)\left[\partial\left(n_{e} k_{\mathrm{B}} T_{e}\right) / \partial r\right]$. The thermal force is dominant in the lower part of the solar corona, where the particles are mainly accelerated (Esser et al. 1997). Moreover, this term is never negligible with respect to the electron-pressure gradient. The importance of the thermal force in the collisional transfer underlines the importance of the suprathermal part in the distribution function. The generalized and biMaxwellian momentum transfers (see Fig. 7, left) are very similar. This is due to the small relative velocity between electrons and protons with respect to the thermal velocity, but also to the small electron-normalized heat flux. The apparent final increase of the transfers is due to the normalization.

The interactions of protons with other species are weak. However, there exists a force, the mirror force, that is rarely considered in the acceleration of the solar wind. This force is related to the proton temperature anisotropy, $T_{p \perp}>T_{p \|}$, in the inner corona (Kohl et al. 1998). This force is equal to $F_{M}=n_{p} k_{\mathrm{B}}\left(T_{p \perp}-T_{p \|}\right)\{[1 / B(r)][\partial B(r) / \partial r]\}$, where $B$ is the magnetic field strength and $r$ is the heliocentric distance (see eq. [A6]). With the parameters described in Figure 6, we are able to evaluate this force between 1 and $10 R_{\odot}$ with respect to the gravity force, equal to $F_{G}=n_{p} m_{p} M_{\odot} G / r^{2}$, where $M_{\odot}$ is the Sun's mass and $G$ is the gravitation constant. In Figure 8 we have plotted the rate $F_{M} / F_{G}$ as a function of the heliocentric distance. We here consider a simple model of $B(r)$ (Pätzold et al. 1987), that is, $B(r)=10^{-4} \times(1.18 /$ $r^{2}+6 / r^{3}$ ) in Tesla. Figure 8 shows that the mirror force is $25 \%$ of the gravity force at $2.5 R_{\odot}$ for a temperature anisotropy of 2 , and this ratio increases with increasing anisotropy and increasing distance $r$. This force tends to accelerate the protons. In this particular case, the proton mirror force is always larger than the electrostatic force, which is equal to $\left[-1 /\left(n_{e} m_{p}\right)\right]\left[\partial\left(n_{e} k_{\mathrm{B}} T_{e}\right) / \partial r\right]$ and which also accelerates the protons. Indeed, the ratio of these two forces is equal to 1.35. For $\mathrm{H} \alpha$ particles, evidence for a temperature anisotropy in the lower corona has not been

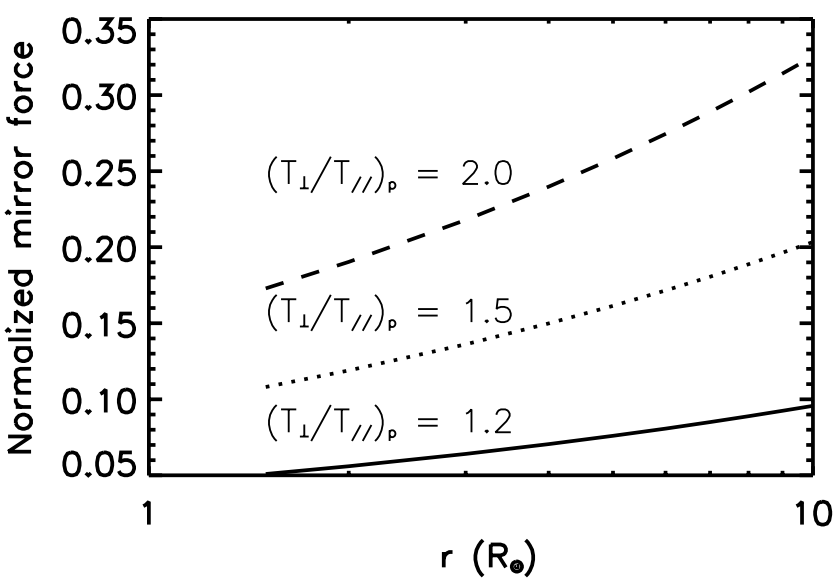

FIG. 8.-Normalized mirror force for the proton as described in $\S 3.2$ and determined for the parameters provided in Fig. 6 . This force is calculated for three different values of the proton temperature anisotropy. 
obtained. For heavier ions, such as $\mathrm{O}^{+5}$, this force could also be important with respect to the gravity force because of a very high temperature anisotropy $\left(T_{\perp} / T_{\|}=10-100\right.$; Kohl et al. 1997, 1998). For electrons, the parallel temperature is usually higher than the perpendicular temperature from 0.3 to $1 \mathrm{AU}$ (Pilipp et al. 1990). The effect of the mirror force is then to slow down the electrons. It should be emphasized that this effect is 30-60 times stronger than the gravity effect between 1.5 and $10 R_{\odot}$ for an electron temperature anisotropy of 1.2. This result must be compared with the electrostatic force, which also slows down the electrons and is equal to $\left[1 /\left(n_{e} m_{e}\right)\right]\left[\partial\left(n_{e} k_{\mathrm{B}} T_{e}\right) / \partial r\right]$. Indeed, this force is 9 times larger than the electron mirror force in the considered case.

Observations of the temperature in the solar corona (Esser 1999; David et al. 1998; Kohl et al. 1996) have shown that the proton temperature is 3-6 times higher than the electron temperature. These differences seem to be higher in the solar corona than at $0.3 \mathrm{AU}$ (Marsch et al. 1982a, 1982b; Pilipp et al. 1990). Therefore, there should be significant effects of thermalization between electrons and protons, which could be the thermalization effects, $m_{e} v_{e p}\left(T_{p}\right.$ $\left.-T_{e}\right) / m_{p}$, and the friction effect, $v_{e p} m_{e}\left(u_{e}-u_{p}\right)^{2} / k_{\mathrm{B}}$. The right panel of Figure 7 shows these two contributions (solid and dashed lines, respectively), but also the correction to the friction term, $2 m_{e} v_{e p}\left(u_{e}-u_{p}\right)\left[\left(-q_{e} / \rho_{e}+q_{p} / \rho_{p}\right) /\left(v_{e p t h}^{2} k_{\mathrm{B}}\right)\right]$ (dash-dotted line) and the generalized total energy transfer (dotted line). The generalized total energy transfer is equal to $\left(\delta p_{e \|} / \delta t+2 \delta p_{e \perp} / \delta t\right) / 2$ (see eqs. [B4] and [B5]). These contributions are normalized to the dominant term in the lefthand part of the equation for the total electron energy, $\left[3 /\left(2 n_{e} k_{\mathrm{B}}\right)\right]\left(\partial q_{e} / \partial r\right)$. According to Figure 7 , the dominant term in the transfer of energy between electrons and protons is the thermalization term from 1.5-2 $R_{\odot}$. At larger heliocentric distances, the thermalization term decreases rapidly, while the friction term and its correction tends to be dominant and to have the same order of magnitude. It is remarkable that in this particular case, the total energy transfer derived from Chodura \& Pohl (1971) is equal to the energy transfer derived from the generalized model. We verified that this result is independent of the value of the electron temperature anisotropy. This means that in the solar corona, the total energy transfer between electrons and protons is composed of a thermalization term, a friction term, and a correction to the friction term. The differences between the generalized energy transfer and the classical thermalization transfer show that the contributions of the heat flux and of the relative velocity in the energy transfer can generate a total energy transfer 1-8 times higher than the thermalization transfer. These differences would generate a less important decrease of the electron temperature in the solar corona than predicted by previous multimoment, multispecies approaches (Olsen \& Leer 1996; Tu \& Marsch 1997; Li et al. 1997).

In order to better understand the nature of the electron distribution function, which is less anisotropic than the proton distribution function and probably not affected by wave-particle interaction, we have analyzed the parallel and perpendicular energy transfers by Coulomb collision with protons. In Figure 9, the left panel presents the parallel energy transfer and the right panel the perpendicular energy transfer. The solid line corresponds to the generalized model and the dotted line to the Chodura \& Pohl (1971) model. The two models predict similar energy transfers for this temperature anisotropy of 1.2. What is remarkable in this figure is that with respect to the right panel of Figure 7, the parallel and perpendicular energy transfers are 1 order of magnitude higher than the total energy transfer. These transfers predict a decrease of the parallel temperature and an increase of the perpendicular temperature. We have found that this result is due to a term in the Chodura \& Pohl (1971) expressions that is proportional to the ratio of the mass of the target particles (protons, in this case) on the mass of the incident particles (electrons). The larger this ratio is, the more important are these transfers that suppress the temperature anisotropy. In conclusion, the electrons become very rapidly isotropic by collisional processes with the protons. Moreover, this situation is amplified by the energy transfer between electrons, which is of the same order of magnitude as the energy transfer between electrons and protons. However, these remarks are valid for a temperature anisotropy equal to 1.2 . For an isotropic electron temperature, the Chodura \& Pohl (1971) model predicts an isotropic energy transfer, whereas the gener-
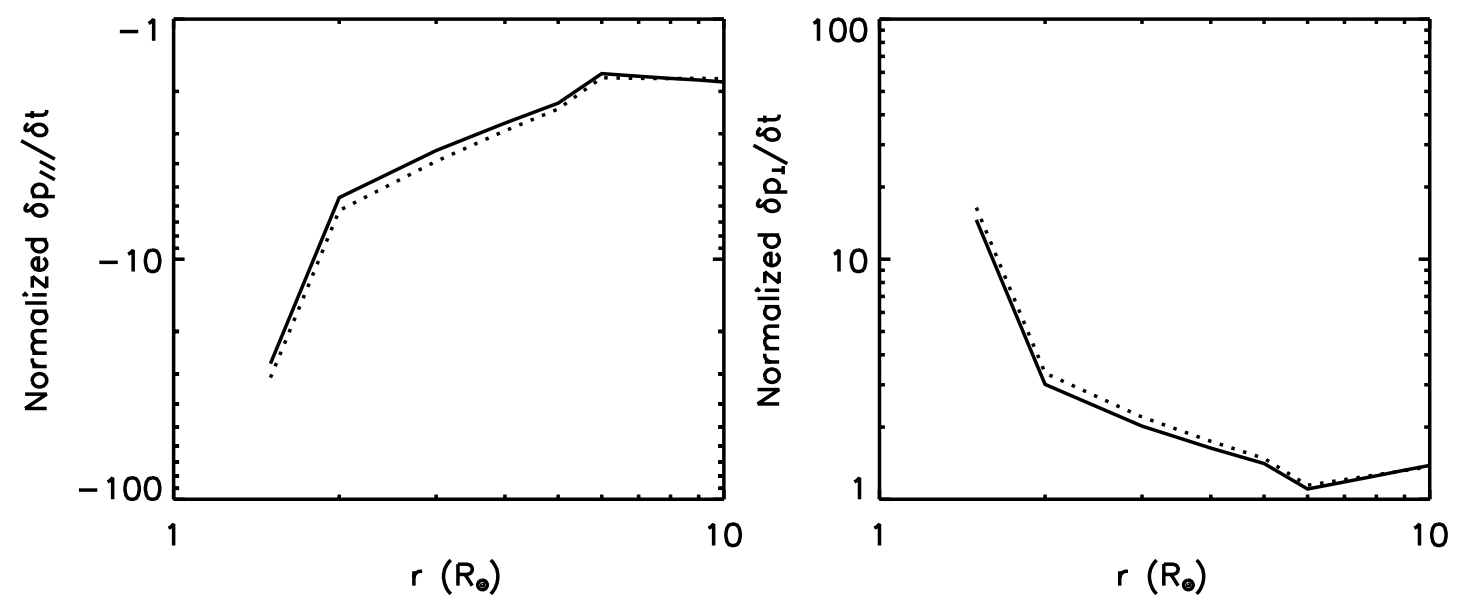

FIG. 9.-Left: Generalized parallel energy transfer (solid line) and bi-Maxwellian parallel energy transfer (dotted line), normalized in the same way as for the right panel of Fig. 7. Right: Same as left panel, but for the perpendicular energy transfer. The parameters used to determined the collisional transfers are those provided in Fig. 6. 
alized model predicts an increase of the parallel energy and a decrease of the perpendicular energy. The total energy transfer is still very similar for both approaches. We think that this difference is due to the assumption that the heat flux is reduced to its component along the parallel direction because of the gyrotropic motion of particles. Indeed, it implies that there are particles in the parallel direction that interact only weakly with the protons, and then that the collisional processes are not efficient enough to suppress the temperature anisotropy. As a consequence, and contrary to the Chodura \& Pohl (1971) model, the generalized energy transfer predicts a small electron temperature anisotropy in the presence of an electron heat flux when only collisions with protons are taken into account. As an example, for a normalized electron heat flux of 0.25 , the temperature anisotropy should stabilize around a value of 1.02. Indeed, it then corresponds to an isotropic energy transfer. Finally, we determined that the main contribution of the parallel and perpendicular energy transfers is the thermalization term, which depends only on the difference between the temperatures of the protons and electrons. This implies that the correction to the friction term, which appears in Figure 7 , is important only for the evaluation of the total energy transfer.

To conclude, the interaction between electrons and protons could partially explain the acceleration of the cold electrons by interaction with the protons, and the decrease of the difference between proton and electron temperatures in the extended corona. There exists in the solar corona a third nonnegligible species: the $\alpha$ ions, whose observed density is around 5\% (Hammer 1997) and highly variable. These are double-charged particles and could also have a significant importance in the process of accelerating the electrons. Moreover, the $\mathrm{H} \alpha$ ions are much hotter than the protons and significantly heat the electrons by thermalization effects and the two other process described above. Indeed, the relative velocity of the $\mathrm{H} \alpha$ ions with respect to the protons is also of the order of the Alfvén speed beyond $0.3 \mathrm{AU}$ (Marsch et al. 1982a) and larger than the proton velocity. Then the relative velocity between the cold electrons and the $\mathrm{H} \alpha$ ions is important.

\section{CONCLUSION}

This is the last of a set of three papers establishing a multimoment, multispecies model for the expansion of astrophysical winds. The first paper (Leblanc \& Hubert 1997) presents the microscopic level of description of the generalized model, while the second paper (Leblanc \& Hubert 1998) presents the macroscopic level, that is, the transport equations of the macroscopic parameters and their properties. In this paper, we complete the macroscopic description by presenting the collisional transfers. We give the generic expressions associated with the six parameters that are necessary to accurately describe the macroscopic evolution of the solar and terrestrial polar winds. We also discuss the approximations made in order to simplify these generic expressions in the context of these winds. Finally, we discuss new aspects of these terms in functions of known collision effects, previous multimoment models (Chodura \& Pohl 1971; Demars \& Schunk 1979), and observations made by the Ulysses, Helios 1 and 2, and $\mathrm{SOHO}$ spacecraft.

By a study relevant to the collisional transition region of the terrestrial polar wind, we have verified the importance of the suprathermal parts of the velocity distribution, which are better described by the generalized model via the heat flux. It must be emphasized that this region is also the region of acceleration and heating of the particles. The transfer of momentum is more important in this region than previously predicted. The energy transfer shows that the heating region is the same as the acceleration region in the terrestrial polar wind. Moreover, the generalized model predicts a new anisotropy of the energy transfers. The transfer of energy is also underestimated by the previous multimoment approaches, especially in the transition region. To sum up, the generalized model of collisional transfers predicts higher transfers in a thinner region between the different species that compose the terrestrial polar wind.

In an application to the solar wind between 1.1 and 10 $R_{\odot}$, in the acceleration region of the fast solar wind, we have also underlined the importance of the suprathermal part of the distribution function. The generalized transfers (Appendix B) and the bi-Maxwellian transfer (Chodura \& Pohl 1971) of momentum and energy are not significantly different for the interaction between electrons and protons. According to the bi-Maxwellian model, such an energy transfer is composed of three contributions: the classical thermalization term, a friction term, and a correction of the friction term. The main contribution beyond $2 R_{\odot}$ is the correction to the friction term. Beyond $2 R_{\odot}$, this contribution generates energy transfer from 1 to 8 times higher than the thermalization term. A study of the anisotropy of the energy transfer has shown that the collisions with protons tend to drastically reduce the electron temperature anisotropy. However, it has also shown that for an electron isotropic temperature and for an electron heat flux reduced to the parallel direction because of the gyrotropic motion of the particles along the magnetic field line, the generalized approach predicts an anisotropic energy transfer between electrons and protons, contrary to the bi-Maxwellian model. The bi-Maxwellian approach predicts isotropic temperature in the presence of an electron heat flux, whereas the generalized approach predicts an anisotropic temperature. In the solar wind, the main contribution of the suprathermal part of the electron velocity distribution function is to enlarge the region where the effects of electron/ proton collision are important. This last remark could help to join the profile of the electron temperature between 1 and $2 R_{\odot}$ observed by $S O H O$ (David et al. 1998) and the profile observed beyond 0.3 AU (Pilipp et al. 1990) more easily. According to this work, despite the very large proton temperature anisotropy measured in the coronal holes (Kohl et al. 1998), the transfer of energy between electrons and protons would maintain a small anisotropic electron temperature. Moreover, an important characteristic of the microscopic state of the fast solar wind is the evolution of the temperature anisotropy of the proton population. We have shown that a moderate temperature anisotropy provides a new force, the mirror force, which represents a significant portion of the gravitational force in the acceleration region. The precise mechanisms originating the proton temperature anisotropy and the region of the solar corona where it takes place is then an important opened question.

Step by step, the properties of the generalized multispecies, multimoment model have been established in an unified approach for the terrestrial polar and solar winds. In this approach, contrary to spectral solutions of the FokkerPlanck equation, the evolution of the different species comprising the winds are described simultaneously and 
self-consistently from collisional to collisionless regions. Many properties of these winds can be described by this approach: the important mean relative velocities between species, the large temperature anisotropy, and the large normalized heat flux. Calculations of the collisional transfers have been analyzed for nonequilibrium states typical of realistic situations. This model provides an opportunity to quantify more accurately pure Coulomb collision effects in shaping velocity distribution, as recently obtained in an application to the terrestrial polar wind (Leblanc et al. 2000). It also offers a starting point for an analysis of the competition between Coulomb collisions and anomalous scattering by wave-particle interaction effects in the field of astrophysical wave-driven winds (Cranmer, Field, \& Kohl 1999).

\section{APPENDIX A}

\section{THE GENERALIZED TRANSPORT EQUATION SYSTEM}

In a gyrotropically dominated situation arising from the presence of a strong magnetic field, the three-dimensional velocity space can be described as a space with two dimensions, the directions parallel and perpendicular to the magnetic field. A vector $B\left(b_{1}, b_{2}, b_{3}\right)$ is then reduced to $B\left(b_{\perp}, b_{\|}\right)$. We also suppose that the motion of the particles is confined along the magnetic field lines. If we consider that the third coordinate corresponds to the direction of the magnetic field, then $\boldsymbol{u}_{s}$ is reduced to $\left(0,0, u_{s}\right)$, only the trace of the tensor $\boldsymbol{P}_{s}$ is nonzero and is equal to $\left(p_{s \perp}, p_{s \perp}, p_{s \|}\right)$, the heat flux of the parallel energy reduces to $\boldsymbol{q}_{s}^{\|}=\left(0,0, q_{s}^{\|}\right)$, and the heat flux of perpendicular energy reduces to $\boldsymbol{q}_{s}^{\perp}=\left(0,0, q_{s}^{\perp}\right)$. The 16 independent parameters of a three-dimensional situation then reduce to the six parameters:

$$
\begin{array}{ll}
n_{s}: & \text { number density } \\
u_{s}=\left\langle v_{s \|}\right\rangle: & \text { species drift velocity in the parallel direction } \\
T_{s \|}=\frac{m_{s}}{k_{\mathrm{B}}}\left\langle c_{s \|}^{2}\right\rangle: & \text { parallel temperature } \\
T_{s \perp}=\frac{m_{s}}{2 k_{\mathrm{B}}}\left\langle c_{s \perp}^{2}\right\rangle: & \text { perpendicular temperature } \\
q_{s}^{\|}=n_{s} m_{s}\left\langle c_{s \|}^{2} c_{s \|}\right\rangle: & \text { heat flux vector for parallel energy in the parallel direction } \\
q_{s}^{\perp}=\frac{1}{2} n_{s} m_{s}\left\langle c_{s \perp}^{2} c_{s \|}\right\rangle: & \text { heat flux vector for perpendicular energy in the parallel direction, }
\end{array}
$$

where $c_{s \perp}$ is the vector $\left(c_{s 1}, c_{s 2}\right), c_{s \|}=c_{s 3}$, and $\rho_{s}=n_{s} m_{s}$ is the mass density. We define a coordinate system where $r$ is the distance in the radial direction from the origin of the system (the heliocentric distance in the case of the solar wind and the geocentric distance in the terrestrial polar wind). We consider a magnetic flux tube radially oriented and spherically symmetric. The coordinate system is reduced to the coordinate $r$ because of the spherical symmetry of the expansion. $A(r)$ is the section of the magnetic flux tube at the distance $r$, which is related to the magnetic field strength, $B(r)$, by the Maxwell law $\partial[A(r) B(r)] / \partial r=0 . G$ is the acceleration due to gravitation, and $E$ is the charge-separation electrostatic field.

For a one-dimensional situation, the velocity distribution function defined in Leblanc \& Hubert (1997) is reduced to

$$
f_{s}\left(r, c_{s}, t\right)=f_{s}^{0}\left(r, c_{s}, t\right)\left[1+\beta_{s \|} \beta_{s \perp}\left(\frac{1}{2} \beta_{s \perp} c_{s \perp}^{2}-1\right) \frac{q_{s \|}^{\perp} c_{s \|}}{n_{s} m_{s}}\right],
$$

where $\beta_{s \perp}=m_{s} / k_{\mathrm{B}} T_{s \perp}$ and $\beta_{s \|}=m_{\mathrm{s}} / k_{\mathrm{B}} T_{s \|}$. The function $f_{s}^{0}$ is the zeroth-order function from which the polynomial part in $c_{s}$ is determined (Mintzer 1965). In order to construct a well-adapted model of VDF for the solar and terrestrial polar winds, we have chosen $f_{s}^{o}$ to be asymmetric along the direction of the magnetic field that corresponds to a large parallel heat flux and a high density of suprathermal particles (Leblanc \& Hubert 1997). This function is defined as

$$
f_{s}^{0}\left(r, c_{s}, t\right)=n_{s} \frac{m_{s}}{4 k_{\mathrm{B}} \pi T_{s \perp} D_{s}^{*}} \exp \left(-\frac{m_{s}}{2 k_{\mathrm{B}} T_{s \perp}} c_{s \perp}^{2}-\frac{c_{s \|}+D_{s}^{*}}{D_{s}^{*}}+\frac{1}{E_{s}^{*}}\right) \operatorname{erfc}\left[E_{s}^{* 1 / 2}\left(\frac{1}{E_{s}^{*}}-\frac{c_{s \|}+D_{s}^{*}}{2 D_{s}^{*}}\right)\right],
$$

where

$$
\begin{aligned}
& D_{s}^{*}=\left(\frac{q_{s}^{\|}}{2 \rho_{s}}\right)^{1 / 3}, \\
& E_{s}^{*}=\frac{2 m_{s} D_{s}^{* 2}}{k_{\mathrm{B}} T_{s^{0}}}, \quad \text { where } \quad T_{s^{0}}=T_{s \|}-\frac{m_{s} D_{s}^{* 2}}{k_{\mathrm{B}}},
\end{aligned}
$$

and erfc is the complementary error function (Abramowitz \& Stegun 1964). 
The set of transport equations for the six parameters (eq. [A1]) is then:

1. Number density equation (for $C_{s}^{p}=1$; see eq. [2]):

$$
\frac{\partial n_{s}}{\partial t}+u_{s} \frac{\partial n_{s}}{\partial r}+n_{s} \frac{\partial u_{s}}{\partial r}+\frac{n_{s} u_{s}}{A} \frac{\partial A}{\partial r}=\frac{\delta n_{s}}{\delta t}
$$

2. Momentum equation (for $C_{s}^{p}=c_{s \|}$; see eq. [2]):

$$
\frac{\partial u_{s}}{\partial t}+u_{s} \frac{\partial u_{s}}{\partial r}+\frac{k_{\mathrm{B}} T_{s \|}}{\rho_{s}} \frac{\partial n_{s}}{\partial r}+\frac{k_{\mathrm{B}}}{m_{s}} \frac{\partial T_{s \|}}{\partial r}+\frac{k_{\mathrm{B}}\left(T_{s \|}-T_{s \perp}\right)}{m_{s}} \frac{1}{A} \frac{\partial A}{\partial r}-\frac{e_{s}}{m_{s}} E-G=\frac{\delta u_{s}}{\delta t} .
$$

3. Parallel temperature equation (associated with $C_{s}^{p}=c_{s \|}^{2}$; see eq. [2]):

$$
\frac{\partial T_{s \|}}{\partial t}+u_{s} \frac{\partial T_{s \|}}{\partial r}+2 T_{s \|} \frac{\partial u_{s}}{\partial r}+\frac{1}{n_{s} k_{\mathrm{B}}} \frac{\partial q_{s}^{\|}}{\partial r}+\frac{q_{s}^{\|}-2 q_{s}^{\perp}}{n_{s} k_{\mathrm{B}}} \frac{1}{A} \frac{\partial A}{\partial r}=\frac{1}{n_{s} k_{\mathrm{B}}} \frac{\delta p_{s \|}}{\delta t}-\frac{T_{s \|}}{n_{s}} \frac{\delta n_{s}}{\delta t}
$$

4. Perpendicular temperature equation (associated with $C_{s}^{p}=c_{s \perp}^{2}$; see eq. [2]):

$$
\frac{\partial T_{s \perp}}{\partial t}+u_{s} \frac{\partial T_{s \perp}}{\partial r}+\frac{1}{n_{s} k_{\mathrm{B}}} \frac{\partial q_{s}^{\perp}}{\partial r}+\left(\frac{2 q_{s}^{\perp}}{n_{s} k_{\mathrm{B}}}+u_{s} T_{s \perp}\right) \frac{1}{A} \frac{\partial A}{\partial r}=\frac{1}{n_{s} k_{\mathrm{B}}} \frac{\delta p_{s \perp}}{\delta t}-\frac{T_{s \perp}}{n_{s}} \frac{\delta n_{s}}{\delta t} .
$$

5. Parallel heat flux equation (associated with $C_{s}^{p}=c_{s \|}^{2} c_{s \|}$; see eq. [2]):

$$
\begin{aligned}
\frac{\partial q_{s}^{\|}}{\partial t}-\frac{m_{s}}{2^{1 / 3}}\left(\frac{q_{s}^{\|}}{\rho_{s}}\right)^{4 / 3} \frac{\partial n_{s}}{\partial r}+4 q_{s}^{\|} \frac{\partial u_{s}}{\partial r}+\frac{3 n_{s} k_{\mathrm{B}}^{2} T_{s \|}}{m_{s}} \frac{\partial T_{s \|}}{\partial r}+ & {\left[u_{s}+2^{5 / 3}\left(\frac{q_{s}^{\|}}{\rho_{s}}\right)^{1 / 3}\right] \frac{\partial q_{s}^{\|}}{\partial r}+u_{s} q_{s}^{\|} \frac{1}{A} \frac{\partial A}{\partial r} } \\
+ & {\left[6 n_{s} m_{s}\left(\frac{q_{s}^{\|}}{2 n_{s} m_{s}}\right)^{4 / 3}-3 \frac{q_{s}^{\|} q_{s}^{\perp}}{n_{s} k_{\mathrm{B}} T_{s \|}}\right] \frac{1}{A} \frac{\partial A}{\partial r}=\frac{\delta q_{s}^{\|}}{\delta t}-3 n_{s} k_{\mathrm{B}} T_{s \|} \frac{\delta u_{s}}{\delta t} }
\end{aligned}
$$

6. Perpendicular heat flux equation (associated with $C_{s}^{p}=c_{s \perp}^{2} c_{s \|}$; see eq. [2]):

$$
\begin{array}{r}
\frac{\partial q_{s}^{\perp}}{\partial t}-\frac{q_{s}^{\|} q_{s}^{\perp}}{n_{s}^{2} k_{\mathrm{B}} T_{s \|}} \frac{\partial n_{s}}{\partial r}+2 q_{s}^{\perp} \frac{\partial u_{s}}{\partial r}-\frac{q_{s}^{\|} q_{s}^{\perp}}{n_{s} k_{\mathrm{B}} T_{s \|}^{2}} \frac{\partial T_{s \|}}{\partial r}+\frac{n_{s} k_{\mathrm{B}}^{2} T_{s \|}}{m_{s}} \frac{\partial T_{s \perp}}{\partial r}+\frac{q_{s}^{\perp}}{n_{s} k_{\mathrm{B}} T_{s \|}} \frac{\partial q_{s}^{\|}}{\partial r}+\left(u_{s}+\frac{q_{s}^{\|}}{n_{s} k_{\mathrm{B}} T_{s \|}}\right) \frac{\partial q_{s}^{\perp}}{\partial r} \\
+2 \frac{q_{s}^{\|} q_{s}^{\perp}}{n_{s} k_{\mathrm{B}} T_{s} \|} \frac{1}{A} \frac{\partial A}{\partial r}+\left[\frac{n_{s} k_{\mathrm{B}}^{2} T_{s \perp}}{m_{s}}\left(T_{s \|}-T_{s \perp}\right)+2 u_{s} q_{s}^{\perp}\right] \frac{1}{A} \frac{\partial A}{\partial r}=\frac{\delta q_{s}^{\perp}}{\delta t}-n_{s} k_{\mathrm{B}} T_{s \perp} \frac{\delta u_{s}}{\delta t}
\end{array}
$$

The terms $\delta n_{s} / \delta t, \delta u_{s} / \delta t, \delta p_{s \|} / \delta t, \delta p_{s \perp} / \delta t, \delta q_{s}^{\|} / \delta t$, and $\delta q_{s}^{\perp} / \delta t$ are the collisional transfers determined from the model of velocity distribution function (eq. [A2]). They are presented in Appendix B.

\section{APPENDIX B}

\section{THE COLLISIONAL TERMS}

In order to present the collisional transfers of the generalized model we must first define

$$
\begin{gathered}
\beta_{s t \|}=\frac{\beta_{s \|} \beta_{t \|}}{\beta_{s \|}+\beta_{t \|}}, \quad \beta_{s t \perp}=\frac{\beta_{s \perp} \beta_{t \perp}}{\beta_{s \perp}+\beta_{t \perp}}, \\
\Gamma_{s}=\frac{3}{4} v_{s t} \frac{\mu_{t}}{\sqrt{\pi}}\left(\frac{\beta_{s t \perp}}{\beta_{s t \|}}\right)^{1 / 2}, \quad \text { where } \quad \mu_{t}=\frac{m_{t}}{m_{s}+m_{t}} .
\end{gathered}
$$

In $\Gamma_{s}, v_{s t}$ is the collision frequency of the particles of the $s$ species with the particles of the $t$ species. 
The expressions of the collisional transfers depend on the following integrals:

$$
\begin{aligned}
V_{2 n 2 m p q}= & \frac{2}{D_{s}^{*} D_{t}^{*} \beta_{s t \mid \|}} \exp \left(\frac{1}{E_{s}^{*}}+\frac{1}{E_{t}^{*}}-2\right) \iiint \int \exp \left[-\frac{\left(2 / \beta_{s t \|}\right)^{1 / 2}}{D_{s}^{*}} x_{s}-\frac{\left(2 / \beta_{s t \|}\right)^{1 / 2}}{D_{t}^{*}} x_{t}-x_{1}^{2}-x_{2}^{2}\right] \\
& \times \operatorname{erfc}\left\{E_{s}^{* 1 / 2}\left[\frac{1}{E_{s}^{*}}-\frac{1}{2}-\frac{\left(2 / \beta_{s t \|}\right)^{1 / 2}}{2 D_{s}^{*}} x_{s}\right]\right\} \operatorname{erfc}\left\{E_{t}^{* 1 / 2}\left[\frac{1}{E_{t}^{*}}-\frac{1}{2}-\frac{\left(2 / \beta_{s t \|}\right)^{1 / 2}}{2 D_{t}^{*}} x_{t}\right]\right\} \\
& \times \frac{x_{1}^{2 n} x_{2}^{2 m} x_{s}^{p} x_{t}^{q}}{\left\{x_{1}^{2}+x_{2}^{2}+\left(\beta_{s t \perp} / \beta_{s t \|}\right)\left[x_{s}-x_{t}+\left(\beta_{s t \mid \|} / 2\right)^{1 / 2}\left(u_{s}-u_{t}\right)\right]^{2}\right\}^{3 / 2}} d x_{1} d x_{2} d x_{s} d x_{t}, \\
V_{n m p q}^{\prime}= & V_{n m p+1 q}-V_{n m p q+1}+\left(\frac{\beta_{s t \|}}{2}\right)^{1 / 2}\left(u_{s}-u_{t}\right) V_{n m p q}, \\
V_{n m p q}^{\prime \prime}= & V_{n m p+2 q}+V_{n m p q+2}+\frac{\beta_{s t \|}}{2}\left(u_{s}-u_{t}\right)^{2} V_{n m p q}+2\left(\frac{\beta_{s t \|}}{2}\right)^{1 / 2}\left(u_{s}-u_{t}\right)\left(V_{n m p+1 q}-V_{n m p q+1}\right)-2 V_{n m p+1 q+1},
\end{aligned}
$$

where $D_{s}^{*}, D_{t}^{*}, E_{s}^{*}$, and $E_{t}^{*}$ are defined in equation (A4). The integral $V_{2 n 2 m p q}$ could be simplified in a two-dimensional integral and could then be easily and accurately determined by numerical methods using an adapted Romberg extrapolation method (Press et al. 1989).

The collisional transfers of the $s$ species that interacts with the $t$ species for the six parameters defined previously (see eq. [A1]) are then:

1. Number density equation: $\delta n_{\mathrm{s}} / \delta t=0$ in the case of the solar wind between $1.1 R_{\odot}$ and several $\mathrm{AU} ; \delta n_{\mathrm{s}} / \delta t$ is equal to the production of $s$ particles due to charge-exchange reaction with neutrals or solar photoionization in the terrestrial polar wind (Blelly \& Schunk 1993).

2. Momentum equation:

$$
\frac{\delta u_{s}}{\delta t}=-\frac{\Gamma_{s}}{4 \mu_{t}}\left(\frac{2}{\beta_{s t \|}}\right)^{1 / 2}\left\{V_{0000}^{\prime}+\beta_{s t \perp}\left(\frac{2}{\beta_{s t \|}}\right)^{1 / 2}\left[\left(2 V_{2010}^{\prime}-V_{0010}^{\prime}\right) \frac{\beta_{s \|} q_{s}^{\perp}}{\rho_{s}}+\left(2 V_{2001}^{\prime}-V_{0001}^{\prime}\right) \frac{\beta_{t \|} q_{t}^{\perp}}{\rho_{t}}\right]\right\} .
$$

3. Parallel energy equation:

$$
\begin{gathered}
F_{s}^{\|}=4 V_{2210}-V_{2010}-\frac{\beta_{s t \perp}}{\mu_{t} \beta_{s t \|}}\left(2 V_{2020}^{\prime}-V_{0020}^{\prime}\right), \\
F_{t}^{\|}=4 V_{2201}-V_{2001}-\frac{\beta_{s t \perp}}{\mu_{t} \beta_{s t \|}}\left(2 V_{2011}^{\prime}-V_{0011}^{\prime}\right), \\
\frac{\delta p_{s \|}}{\delta t}=\frac{\rho_{s} \Gamma_{s}}{\beta_{s t \perp}}\left\{V_{2000}-\frac{1}{\mu_{t}} \frac{\beta_{s t \perp}}{\beta_{s t \|}} V_{0010}^{\prime}+\beta_{s t \perp}\left(\frac{2}{\beta_{s t \|}}\right)^{1 / 2}\left(F_{s}^{\|} \frac{\beta_{s \|} q_{s}^{\perp}}{\rho_{s}}+F_{t}^{\|} \frac{\beta_{t \|} q_{t}^{\perp}}{\rho_{t}}\right)\right\} .
\end{gathered}
$$

4. Perpendicular energy equation:

$$
\begin{aligned}
& F_{s}^{\perp}=4 V_{2210}-V_{2010}+\frac{\beta_{s t \perp}}{\beta_{s t \|}}\left(2 V_{2010}^{\prime \prime}-V_{0010}^{\prime \prime}\right)+\frac{2}{\mu_{t}}\left[\left(\frac{\beta_{s t \perp}}{\beta_{s \perp}}-\frac{\beta_{s t \perp}}{\beta_{t \perp}}\right) V_{2010}-4 \frac{\beta_{s t \perp}}{\beta_{s \perp}} V_{2210}\right], \\
& F_{t}^{\perp}=4 V_{2201}-V_{2001}+\frac{\beta_{s t \perp}}{\beta_{s t \|}}\left(2 V_{2001}^{\prime \prime}-V_{0001}^{\prime \prime}\right)+\frac{4}{\mu_{t}} \frac{\beta_{s t \perp}}{\beta_{s \perp}}\left(-2 V_{2201}+V_{2001}\right), \\
& \frac{\delta p_{s \perp}}{\delta t}=\frac{\rho_{s} \Gamma_{s}}{2 \beta_{s t \perp}}\left[\left(1-\frac{2 \beta_{s t \perp}}{\mu_{t} \beta_{s \perp}}\right) V_{2000}+\frac{\beta_{s t \perp}}{\beta_{s t \|}} V_{0000}^{\prime \prime}+\beta_{s t \perp}\left(\frac{2}{\beta_{s t \|}}\right)^{1 / 2}\left(F_{s}^{\perp} \frac{\beta_{s\|\|} q_{s}^{\perp}}{\rho_{s}}+F_{t}^{\perp} \frac{\beta_{t\|\|} q_{t}^{\perp}}{\rho_{t}}\right)\right] .
\end{aligned}
$$

5. Parallel heat flux equation:

$$
\begin{gathered}
H_{s 3}^{\|}=8 V_{2220}-2 V_{2020}+\frac{1}{\mu_{t}} \frac{\beta_{s t \perp}}{\beta_{s t \|}}\left(V_{0030}^{\prime}-2 V_{2030}^{\prime}\right), \\
H_{t 3}^{\|}=8 V_{2211}-2 V_{2011}+\frac{1}{\mu_{t}} \frac{\beta_{s t \perp}}{\beta_{s t \|}}\left(V_{0021}^{\prime}-2 V_{2021}^{\prime}\right), \\
\frac{\delta q_{s}^{\|}}{\delta t}=\frac{3}{2} \frac{\rho_{s} \Gamma_{s}}{\beta_{s t \perp}}\left(\frac{2}{\beta_{s t \|}}\right)^{1 / 2}\left[2 V_{2010}-\frac{1}{\mu_{t}} \frac{\beta_{s t \perp}}{\beta_{s t \|}} V_{0020}^{\prime}+\beta_{s t \perp}\left(\frac{2}{\beta_{s t \|}}\right)^{1 / 2}\left(H_{s 3}^{\|} \frac{\beta_{s \|} q_{s}^{\perp}}{\rho_{s}}+H_{t 3}^{\|} \frac{\beta_{t \|} q_{t}^{\perp}}{\rho_{t}}\right)\right] .
\end{gathered}
$$


6. Perpendicular heat flux equation:

$$
\begin{aligned}
G_{s 3}^{\perp}= & 4 V_{2220}-V_{2020}+\frac{\beta_{s t \perp}}{\beta_{s t \|}}\left(2 V_{2020}^{\prime \prime}-V_{0020}^{\prime \prime}\right)+2 \frac{\beta_{s t \perp}}{\beta_{s \perp}}\left[\left(1-\frac{\beta_{s \perp}}{\beta_{t \perp}}\right) V_{2010}^{\prime}-4 V_{2210}^{\prime}\right] \\
+ & \frac{2}{\mu_{t}} \frac{\beta_{s t \perp}}{\beta_{s \perp}}\left[-4 V_{2220}+\left(1-\frac{\beta_{s \perp}}{\beta_{t \perp}}\right) V_{2020}\right]+\frac{1}{\mu_{t}}\left(\frac{\beta_{s t \perp}}{\beta_{s \perp}}\right)^{2}\left[-4 V_{2210}^{\prime}+\left(1-3 \frac{\beta_{s \perp}}{\beta_{t \perp}}\right) V_{2010}^{\prime}+\frac{\beta_{s \perp}}{\beta_{t \perp}}\left(1-\frac{\beta_{s \perp}}{\beta_{t \perp}}\right) \frac{V_{0010}^{\prime}}{2}\right], \\
G_{t 3}^{\perp}= & 4 V_{2211}-V_{2011}+\frac{\beta_{s t \perp}}{\beta_{s t \|}}\left(2 V_{2011}^{\prime \prime}-V_{0011}^{\prime \prime}\right)+4 \frac{\beta_{s t \perp}}{\beta_{s \perp}}\left(V_{2001}^{\prime}-2 V_{2201}^{\prime}\right)+\frac{4}{\mu_{t}} \frac{\beta_{s t \perp}}{\beta_{s \perp}}\left(-2 V_{2211}+V_{2011}\right) \\
+ & \frac{1}{\mu_{t}}\left(\frac{\beta_{s t \perp}}{\beta_{s \perp}}\right)^{2}\left[-4 V_{2201}^{\prime}+\left(3-\frac{\beta_{s \perp}}{\beta_{t \perp}}\right) V_{2001}^{\prime}-\left(1-\frac{\beta_{s \perp}}{\beta_{t \perp}}\right) \frac{V_{0001}^{\prime}}{2}\right], \\
\frac{\delta q_{s}^{\perp}}{\delta t}= & \frac{3}{2} \frac{\rho_{s} \Gamma_{s}}{\beta_{s t \perp}}\left(\frac{2}{\beta_{s t \|}}\right)^{1 / 2}\left[-\frac{1}{\mu_{t}} \frac{\beta_{s t \perp}}{\beta_{s \perp}}\left(\frac{\beta_{s t \perp}}{\beta_{t \perp}} \frac{V_{0000}^{\prime}}{2}+\frac{\beta_{s t \perp}}{\beta_{s \perp}} V_{2000}^{\prime}+2 V_{2010}\right)\right. \\
& \left.+V_{2010}-2 \frac{\beta_{s t \perp}}{\beta_{s \perp}} V_{2000}^{\prime}+\frac{\beta_{s t \perp}}{\beta_{s t \|}} V_{0010}^{\prime \prime}+\beta_{s t \perp}\left(\frac{2}{\beta_{s t \|}}\right)^{1 / 2}\left(G_{s 3}^{\perp} \frac{\beta_{s \|}}{\rho_{s}}+q_{t 3}^{\perp} \frac{\beta_{t \|} q_{t}^{\perp}}{\rho_{t}}\right)\right] .
\end{aligned}
$$

\section{REFERENCES}

Abe, H., et al. 1993, J. Geophys. Res., 98, 11191

Abramowitz, M., \& Stegun, I. A. 1964, Handbook of Mathematical Functions (New York: Dover)

Banks, P. M., \& Holzer, T. E. 1968, J. Geophys. Res., 73, 6846

Bell, A. R., Evans, R. G., \& Nicholas, D. J. 1980, Phys. Rev. Lett., 46, 243

Blelly, P. L., \& Schunk, R. W. 1993, Ann. Geophys., 11, 443

Burgers, J. M. 1969, Flow Equations for Composite Gases (New York: Academic Press)

Chamberlain, J. W. 1960, ApJ, 131, 47

Chodura, R., \& Pohl, F. 1971, Plasma Phys., 13, 645

Cranmer, S. R., Field, G. B., \& Kohl, J. L. 1999, ApJ, 518, 937

David, C., et al. 1998, A\&A, 336, L90

Demars, H. G., \& Schunk, R. W. 1979, J. Phys. D, 12, 1501

Esser, R., Fineschi, S., Dobrzycka, D., Habbal, S. R., Edgar, R. J., Raymond, J. C., \& Kohl, J. L. 1999, ApJ, 510, L63

Esser, R., Habbal, S. R., Coles, W. A., \& Hollweg, J. V. 1997, J. Geophys. Res., 102, 7063

Esser, R., Richard, J. E., \& Brickhouse, N. S. 1998, ApJ, 498, 448

Feldman, W. C., Asbridge, J. R., Bame, S. J., Gary, S. P., Montgomery, M. D., \& Zink, S. M. 1976, J. Geophys. Res., 81, 5207

Ganguli, S. B. 1996, Rev. Geophys., 34, 311

Grad, H. 1958, in Principles of the Kinetic Theory of Gases, ed. S. Flügge (Berlin: Springer), 205

Hammer, R. 1997, in Fifth SOHO Workshop: The Corona and Solar Wind Near Minimum Activity, ed. A. Wilson (Noordwijk: ESA), 141

Issautier, K., Meyer-Vernet, N., Moncuquet, M., \& Hoang, S. 1998, J. Geophys. Res., 103, 1969

Jancel, R., \& Kahan, J. 1963, Electrodynamique des Plasmas (Paris: Dunod)
Jones, G. O. L., Williams, P. J. S., Winser, K. J., Lockwood, M., \& Suvanto, K. 1998, Nature, 336, 231

Kohl, J. L., Strachan, L., \& Gardner, L. D. 1996, ApJ, 465, L141

Kohl, J. L., et al. 1997, Sol. Phys., 175, 613

. 1998, ApJ, 501, L127

Leblanc, F., \& Hubert, D. 1997, ApJ, 483, 464

. 1998, ApJ, 501, 375; erratum 511, 515 (1999)

Leblanc, F., Hubert, D., \& Blelly, P.-L. 2000, J. Geophys. Res., in press

Lemaire, J., \& Scherer, M. 1973, Rev. Geophys. Space Phys., 11, 427

Li, X. 1999, J. Geophys. Res., in press

Li, X., Esser, R., \& Habbal, S. R. 1997, J. Geophys. Res., 102, 17419

Marsch, E., Mühlhäuser, K. H., Rosenbauer, H., Schwenn, R., \& Neubauer, F. M. 1982a, J. Geophys. Res., 87, 35

Marsch, E., Mühlhäuser, K. H., Schwenn, R., Rosenbauer, H., Pilipp, W., \& Neubauer, F. M. 1982b, J. Geophys. Res., 87, 52

Mintzer, D. 1965, Phys. Fluids, 8, 1076

Olsen, E. L., \& Leer, E. 1996, J. Geophys. Res., 101, 15591

. 1999, J. Geophys. Res., 104, 9963

Oraevskii, V., Chodura, R., \& Feneberg, W. 1968, Plasma Phys., 10, 819

Parker, E. N. 1958, ApJ, 128, 664

Pätzold, M., et al. 1987, Sol. Phys., 109, 91

Pilipp, W. G., et al. 1990, J. Geophys. Res., 95, 6305

Press, W. H., Flannery, B. P., Teukolsky, S. A., \& Vetterling, W. T. 1989, Numerical Recipes (Cambridge: Cambridge Univ. Press)

Schunk, R. W. 1977, Rev. Geophys. Space Phys., 15, 429

Scime, E. E., Bame, S. J., Feldman, W. C., Gary, S. P., \& Phillips, J. L. 1994, J. Geophys. Res., 99, 23401

Tanenbaum, B. S. 1967, Plasma Phys. (New York: McGraw Hill)

Tu, C.-Y., \& Marsch, E. 1997, Sol. Phys., 171, 363 